\title{
Dynamics of coronal mass ejections in the interplanetary medium
}

\author{
A. Borgazzi ${ }^{1,2}$, A. Lara $^{2}$, E. Echer ${ }^{1}$, and M. V. Alves ${ }^{1}$ \\ 1 National Institute for Space Research, INPE, SP-Brazil \\ e-mail: andrea@dge.inpe.br \\ 2 Instituto de Geofísica, Universidad Nacional Autónoma de México, México \\ e-mail: alara@geofisica.unam.mx
}

Received 16 October 2008 / Accepetd 13 February 2009

\section{ABSTRACT}

\begin{abstract}
Context. Coronal mass ejections (CMEs) are large plasma structures expelled from the low corona to the interplanetary space with a wide range of speeds. In the interplanetary medium CMEs suffer changes in their speeds because of interaction with the ambient solar wind.

Aims. To understand the interplanetary CME (ICME) dynamics, we analyze the interaction between these structures and the ambient solar wind (SW), approaching the problem from the hydrodynamic point of view.

Methods. We assume that the dynamics of the system is dominated by two kinds of drag-force dependence on speed $(U)$, as $\sim U$ and $\sim U^{2}$. Furthermore, we propose a model that takes variations of the ICME radius $(R)$ and SW density $\left(\rho_{\text {sw }}\right)$ into account as a function of the distance $(x)$ as $R(x)=x^{0.78}$ and $\rho_{\mathrm{sw}}(x)=1 / x^{2}$, respectively. Then, we solve the equation of motion and present exact solutions Results. Considering CME speeds measured at a few solar radii and at one AU, we were able to constrain the values of the constants (viscosity and drag coefficient) for the linear $(U)$ and quadratic $\left(U^{2}\right)$ speed dependences, which seems to reproduce the ICME - SW system well. We found different solutions in which the concavity of the curves of the ICME speed profile changes, depending on the dominant factor, either the ICME radius or the SW density.

Conclusions. This work shows that the macroscopic ICME propagation may be described by the hydrodynamic theory and that it is possible to find analytical solutions for the ICME-SW interaction.
\end{abstract}

Key words. hydrodynamics - Sun: coronal mass ejections (CMEs) - interplanetary medium

\section{Introduction}

A major solar transient event that injects large amounts of mass and energy to the interplanetary space is known as coronal mass ejection (CME). These vast structures of plasma and magnetic field, observed principally with white light coronographs, are known as the main link between the Sun's activity and geomagnetic storms (Gosling et al. 1999). We classify CMEs according to their initial speed into two categories: "slow" for CMEs with speed lower than $400 \mathrm{~km} \mathrm{~s}^{-1}$; and "fast" for CMEs with speeds higher than $400 \mathrm{~km} \mathrm{~s}^{-1}$. In both cases, an interaction between the ICME structure (the remnant of a CME in the interplanetary medium) and the ambient solar wind takes place. This interaction can be described as a momentum transfer involving two systems, a body (the CME) moving in an ambient fluid, the solar wind $(\mathrm{SW})$. In the case of "fast" CMEs the process causes a deceleration of the ICME structure from its initial speed (in the range of $400 \mathrm{~km} \mathrm{~s}^{-1}$ to $3000 \mathrm{~km} \mathrm{~s}$ ) towards the value of the SW speed $(400 \mathrm{~km} \mathrm{~s})$ at distances of a few AU. In general, the ICME dynamics have been deduced by complementary observations (of the same event) close to the Sun by coronographs and close to the Earth by satellite in-situ measurements. This restriction (observations from only two points) has been overcome by the interplanetary scintillation (IPS) technique (Manoharan 2006) and direct white-light observations in the interplanetary medium (Tappin 2004).

Using these observational methods, the acceleration of ICMEs has been confirmed; however, the profile and mechanisms of the ICME dynamics are still unknown. Many efforts have been made to describe the dynamics of ICMEs. Different kinds of theoretical, empirical, and numerical models have been studied. Recent analytical models of ICME propagation have been presented by Canto et al. (2005) and Borgazzi et al. (2008, hereafter Paper 1). Canto et al. (2005) studied the dynamics of a fluctuation in density and speed starting at the base of the solar corona and traveling in the ambient solar wind. They used a kinematic approximation, proposed CME properties as velocity, density, and temperature, and found the ICME travel time from a few solar radii $\left(R_{\odot}\right)$ to one AU. In Paper 1 , the authors found exact solutions for the behavior of the speed versus time of ICMEs. The interaction between the ICME and the surrounding medium is described by the action of viscous forces, and values of drag and viscous coefficients for the medium were obtained. Empirical models were developed mainly to forecast the $1 \mathrm{AU}$ arrival time of ICMEs (e.g. Gopalswamy et al. 2000, 2001, 2005). The methodology applied in these works consists on the establishment of relationships between the observed data, i.e., the CME initial speed; speeds observed in the interplanetary medium; and the mean acceleration acting on the ICMEs. The studies reported by Vršnak (2001); Vršnak et al. (2002, 2004, 2007) may be considered in the same branch, with the difference that in these cases the studies are done, from the point of view of viscous forces acting on the ICMEs. The group of numerical simulation of the transport of ICMEs is more numerous (e.g. Vandas et al. 1995; Cargill et al. 1996; Cargill 2004; Odstrčil et al. 1999a,b; Gonzalez-Esparza et al. 2003; 
Chen 1996). They generally study the evolution, through the interplanetary space, of some properties (temperature, density and speed) of the plasma structure (plasmoids or magnetic clouds).

To better understand the ICME behavior and to encourage this kind of work (from a theoretical point of view), in this work we approach the study of the ICMEs dynamics using the hydrodynamics theory and considering the ICME - SW system as two interacting fluids, under the action of viscous forces without taking the microscopic details of this interaction into account.

\section{Dynamical propagation model of ICMEs}

\subsection{Initial ICME conditions}

In this work we concentrate in the ICME dynamics, from 30 to $\sim 215 R_{\odot}$. As the initial point is far enough from the solar surface, we assume the following considerations, presented by Chen (1996) and Sheeley et al. (1997):

- the gravity force is negligible. For example, in Fig. 7 of Chen (1996) the gravity force tends to zero at approximately 110 min after CME initiation, corresponding to a distance of the expanding loop apex of $\sim 2 R_{\odot}$ (see Fig. 5 of the same paper);

- the Lorentz force is negligible. In Fig. 9 of Chen (1996), the Lorentz force is practically zero after $200 \mathrm{~min}$ of the CME initiation. This time corresponds to an apex height of $\sim 6 R_{\odot}$;

- the solar wind speed is constant. At $30 R_{\odot}$ the solar wind has already been formed. We assume a constant speed of $400 \mathrm{~km} \mathrm{~s}^{-1}$. Observationally, Sheeley et al. (1997) found that this constant speed begins at approximately $30 R_{\odot}$. Some models assume a speed profile of the form $v_{\mathrm{sw}}=$ $a_{0} \tanh \left(x / b_{0}\right)$ and adjust the constants $a_{0}$ and $b_{0}$ to obtain a constant speed $\left(400 \mathrm{~km} \mathrm{~s}^{-1}\right)$ at a given distance, for example at $35 R_{\odot}$ (Chen 1993) and $40 R_{\odot}$ (Chen 1996);

- after the initial point, the drag force is the only force acting on the system;

- We do not consider the magnetic properties of ICMEs.

\subsection{Basic theory}

When a body moves in a fluid, a force starts acting over the body due to the interaction with the surrounding medium. This force is due to the relative motion between the body and the fluid and is named drag force in a general way. In a first approach we can distinguish between two types of drag forces, one that has a linear dependence on the velocity, whereas another has a quadratic dependence (Kundu \& Cohen 2004). In our approach, the body is the ICME and the SW is the ambient fluid. The main concern in this model may be that we are only considering the hydrodynamic behavior of the system, i.e., no magnetic field interaction is considered. As the plasma in the interplanetary medium has a very low density and is collisionless, the momentum transfer may be due by waves or other collective microscopic processes that, in this approximation, are not relevant. For a macroscopic quantitative description of the ICME - SW dynamics involving drag force, we must take the equation of motion for incompressible fluids $(\nabla \cdot \boldsymbol{u}=0)$ into account, i.e., the Navier-Stokes equation:

$\rho \frac{D \mathbf{u}}{D t}=-\nabla p+\rho \mathbf{g}+\mu \nabla^{2} \mathbf{u}$

where $\rho$ is the ambient density, $\boldsymbol{u}$ is the velocity of the fluid, $p$ the pressure, and $\mu$ the viscosity coefficient. Considering stationary conditions for the flow and neglecting gravity, we can rewrite the last equation as

$\rho \boldsymbol{u} \cdot \nabla \boldsymbol{u}=-\nabla p+\mu \nabla^{2} \boldsymbol{u}$.

A useful parameter in hydrodynamics theory is the Reynolds number

$\mathfrak{R}=\frac{\rho d U}{\mu}$

where $d$ is a characteristic length of the body, $U$ the speed, and $\mu$ the viscosity of the medium. In general, $\mathfrak{R}$ is used to describe the behavior of the system and, in general, two extreme cases are considered: when the speed is dominant $(\mathfrak{R} \gg 1)$ or when the viscosity is dominant $(\mathfrak{R} \ll 1)$. In the case of high Reynolds number $(\mathfrak{R} \gg 1)$, Eq. (2) can be written as (Kundu \& Cohen 2004):

$\rho \boldsymbol{u}^{\prime} \cdot \nabla \mathbf{u}^{\prime}=-\nabla p^{\prime}+\frac{1}{\mathfrak{R}} \nabla^{2} \boldsymbol{u}^{\prime}$.

On the other hand, for low Reynolds number ( $\mathfrak{1} \ll 1)$, Eq. (2), takes the form

$\mathfrak{R} \boldsymbol{u}^{\prime} \cdot \nabla \boldsymbol{u}^{\prime}=-\nabla p^{\prime}+\nabla^{2} \boldsymbol{u}^{\prime}$.

From Eqs. (4) and (5), it is possible to derive the expressions for the force acting on a body in a "laminar" (low $\mathfrak{R}$ ) or "turbulent" (high $\mathfrak{R}$ ) regimes (see Kundu \& Cohen 2004, for details) as

$F_{l}=6 \pi \mu R \cdot U$

and

$F_{t}=\frac{C_{\mathrm{d}} A \rho_{\mathrm{sw}} \cdot U^{2}}{2}$

In Eqs. (6) and (7), the indexs $l$ and $t$ refers to laminar and turbulent regime, $\mu$ is the viscosity of the medium, $R$ the ICME radius, $\rho_{\mathrm{sw}}$ the density of the interplanetary medium, and $A$ the cross section of the ICME that is in contact with the interplanetary medium (considering spherical symmetry). A common dimensionless parameter $C_{\mathrm{d}}$ describes the "intensity" of the drag force. It depends mainly on the shape of the body and could be defined as $C_{\mathrm{d}}=F_{t} /\left(\frac{1}{2} \rho U^{2} A\right)$, i. e. the rate between the drag force and the kinetic energy density and the transversal area of the body (Landau 1987). It is useful to describe the $C_{\mathrm{d}}$ parameter as a function of the Reynolds number (Eq. (3)). For example, for a spherical body, the drag coefficient is between 0.4 and 0.6 ., in the high Reynolds number range (between $10^{3}$ and $10^{5}$ ), whereas for a low Reynolds number $\left(2 \times 10^{-1}\right)$ the $C_{\mathrm{d}}$ is $\sim 10^{2}$ (see Fig. 10.10, Daugherty et al. 1989). Therefore, the value of $C_{\mathrm{d}}$ depends on the system variables and is difficult to give absolute numbers in the ICME context. The ICME deceleration described by our model involves the action of Eqs. (6) and (7), as was described in Paper 1. We note that both forces, "laminar" and "turbulent" are used in similar ways as in the hydrodynamics theory.

\subsection{Variability of ICME radius}

We used the expressions for the drag forces (Eqs. (6) and (7)) in the equation of motion. We also consider that the ICME radius $(R)$ varies with the distance as $R=x^{p}$, assuming $p=0.78$ (Liu et al. 2005). In a system of reference where the ambient $\mathrm{SW}$ is at rest, and therefore, the ICME speed is $U=U_{\mathrm{cme}}-U_{\mathrm{sw}}$, where $U_{\mathrm{sw}}$ is the solar wind speed, we obtain the following differential equations for "laminar" and "turbulent" regimes:

$-6 \pi \mu x^{p}\left(U-U_{\mathrm{sw}}\right)=m_{\mathrm{cme}} U \cdot \frac{\mathrm{d} U}{\mathrm{~d} x}$ 


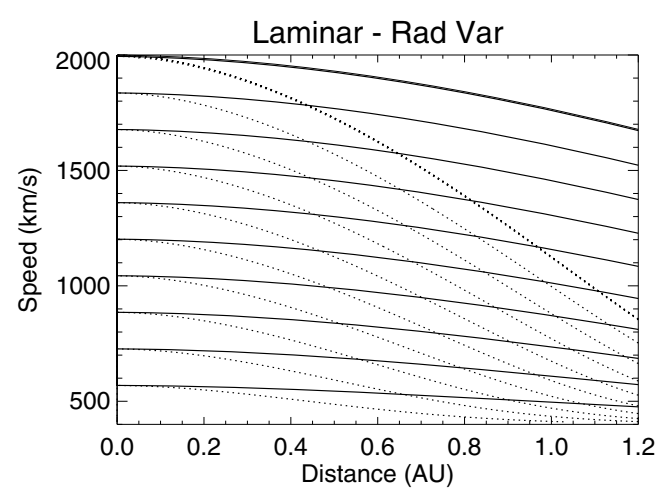

Fig. 1. ICME speed versus distance for "laminar regime", showing a weak ( $\mu=0.1 \mathrm{~g} / \mathrm{cm} \mathrm{s}$ in continuous lines) and strong $(\mu=0.25 \mathrm{~g} / \mathrm{cm} \mathrm{s}$ in dotted lines) ICME - SW interaction. Here and in the following figures, the $y$-axis indicates the initial CME speed used to obtain the presented curves.

and

$$
-\frac{C_{\mathrm{d}} \pi \rho_{\mathrm{sw}} x^{2 p}}{2}\left(U-U_{\mathrm{sw}}\right)^{2}=m_{\mathrm{cme}} U \cdot \frac{\mathrm{d} U}{\mathrm{~d} x} .
$$

Solutions of Eqs. (8) and (9) are given by

$$
\begin{aligned}
x^{(p+1)}-x_{0}^{(p+1)}= & -\frac{m_{\mathrm{cme}}(p+1)}{6 \pi \mu} \\
& \times\left(U-U_{0}+U_{\mathrm{sw}} \ln \left[\frac{\left(U-U_{\mathrm{sw}}\right)}{\left(U_{0}-U_{\mathrm{sw}}\right)}\right]\right)
\end{aligned}
$$

and

$$
\begin{aligned}
& x^{(2 p+1)}-x_{0}^{(2 p+1)}=-2 \frac{m_{\mathrm{cme}}(2 p+1)}{C_{\mathrm{d}} \pi \rho_{\mathrm{sw}}} \\
& \quad \times\left[\frac{U_{\mathrm{sw}}}{\left(U_{0}-U_{\mathrm{sw}}\right)}-\frac{U_{\mathrm{sw}}}{\left(U-U_{\mathrm{sw}}\right)}+\ln \left[\frac{\left(U-U_{\mathrm{sw}}\right)}{\left(U_{0}-U_{\mathrm{sw}}\right)}\right]\right] .
\end{aligned}
$$

In Eqs. (10) and (11), $U_{0}$ and $x_{0}$ are the initial CME speed and position (as measured at the coronograph field of view). To analyze the motion described by Eqs. (10) and (11), we present in Figs. 1 and 2 the resulting speed as a function of the position, for different initial speeds, considering $U_{\mathrm{sw}}=400 \mathrm{~km} \mathrm{~s}^{-1}$. The plots are for two different values of $\mu=0.1 \mathrm{~g} / \mathrm{cm} \mathrm{s}$ (continuous lines) and $\mu=0.25 \mathrm{~g} / \mathrm{cm} \mathrm{s}$ (dotted lines); and $C_{\mathrm{d}}=6 \times 10^{4}$ (continuous lines) and $C_{\mathrm{d}}=1.6 \times 10^{5}$ (dotted lines). In all cases, we have chosen the parametres $\left(\mu\right.$ and $\left.C_{\mathrm{d}}\right)$ in such a way that the ICME speed at $1 \mathrm{AU}$ fall in a range similar to the observed (see Paper 1 for more details).

\subsection{Density variation in the interplanetary medium}

To improve the analysis, we used the Leblanc et al. (1996) model to account for the variation in the SW density with the distance, given by

$\rho_{\mathrm{sW}}(x)=\frac{a}{x^{2}}+\frac{b}{x^{4}}+\frac{c}{x^{6}}$,

where $a=3.3 \times 10^{5}, b=4.1 \times 10^{6}$ and $c=8.0 \times 10^{7}$ are empirical coefficients. The quadratic term represent the density variation in the interplanetary space, whereas the high-order terms represent the density variation in the low corona. In this way, the parameter $a$ is computed using the density measured at 1 AU. As we are interested only on the density variation in the interplanetary medium (from $30 R_{\odot}$ to $215 R_{\odot}$ ), we only take the quadratic term in Eq. (12) into account.

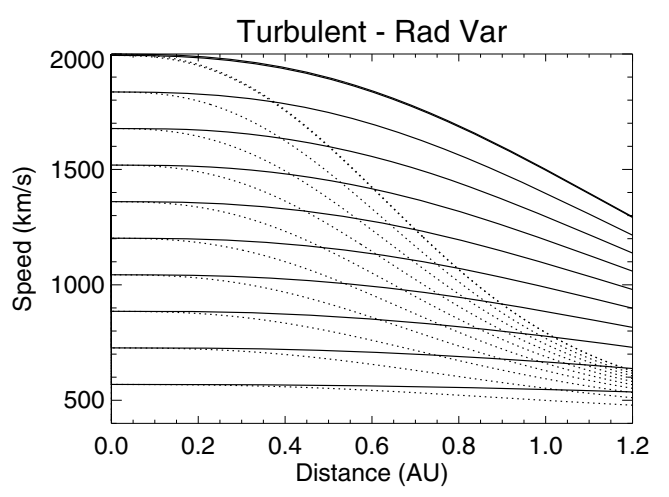

Fig. 2. ICME speed versus distance for "turbulent regime", showing a weak $\left(C_{\mathrm{d}}=6 \times 10^{4}\right.$ in continuous lines $)$ and strong $\left(C_{\mathrm{d}}=1.6 \times 10^{5}\right.$ in dotted lines) ICME - SW interaction.

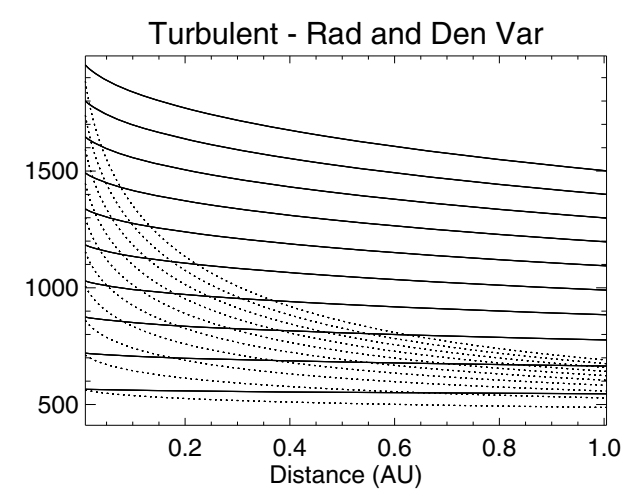

Fig. 3. ICME speed versus distance for "turbulent regime" considering ICME radius and SW density variable. The ICME - SW interaction is weak for a drag coefficient $C_{\mathrm{d}}=2 \times 10^{4}$ (continuous lines) and strong for $C_{\mathrm{d}}=8 \times 10^{4}$ (dotted lines).

\subsection{1. "Turbulent" regime}

The "turbulent" regime, described by Eq. (7), has an explicit dependence with the density, therefore, by using $\rho_{\mathrm{sw}}=a / x^{2}$ we obtain the following equation

$$
-\frac{C_{\mathrm{d}} \pi a x^{(2 p-2)}}{2}\left(U-U_{\mathrm{sw}}\right)^{2}=m_{\mathrm{cme}} U \cdot \frac{\mathrm{d} U}{\mathrm{~d} x} .
$$

The solution is given by

$$
\begin{aligned}
-\frac{C_{\mathrm{d}} \pi a}{2 m_{\mathrm{cme}}(2 p-1)} & {\left[x^{(2 p-1)}-x_{0}^{(2 p-1)}\right]=\frac{U_{\mathrm{sw}}}{\left(U_{0}-U_{\mathrm{sw}}\right)} } \\
& -\frac{U_{\mathrm{sw}}}{\left(U-U_{\mathrm{sw}}\right)}+\ln \left[\frac{\left(U-U_{\mathrm{sw}}\right)}{\left(U_{0}-U_{\mathrm{sw}}\right)}\right] .
\end{aligned}
$$

The behavior of the speed versus position, given by Eq. (14), can be seen in Fig. 3, where we have plotted the solutions for different initial speeds and using two drag coefficients, $2 \times 10^{4}$ (continuous lines) and $8 \times 10^{4}$ (dotted lines).

\subsection{2. "Laminar" regime}

To describe the motion of the ICMEs considering the "laminar" regime, it is necessary to express the "laminar" force as a function of the density of the medium (in this case the SW density). Therefore, we use the relationship between the dynamic 


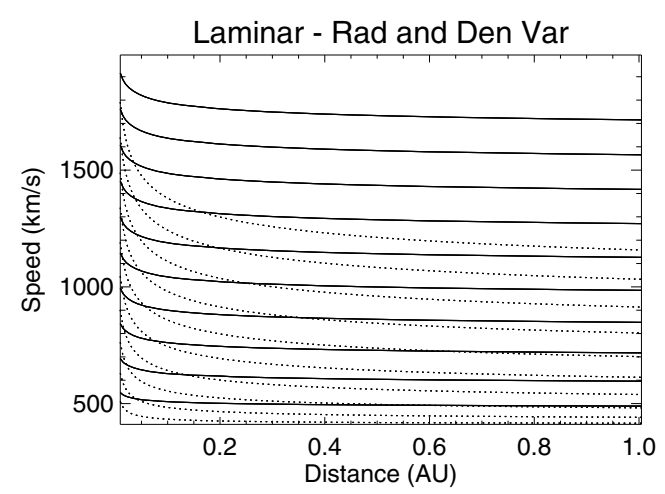

Fig. 4. ICME speed versus distance for "laminar regime" considering ICME radius and SW density variable, in this case the value of the kinematic viscosity is $5 \times 10^{20} \mathrm{~cm}^{2} / \mathrm{s}$ for the weak ICME - SW interaction (continuous lines) and $1.25 \times 10^{21} \mathrm{~cm}^{2} / \mathrm{s}$ for the strong interaction (dotted lines).

viscous coefficient $\mu$ and the kinematic viscous coefficient $v$, as (see Kundu \& Cohen 2004, for details):

$v=\frac{\mu}{\rho_{\mathrm{sw}}}$.

Using Eq. (15), the equation of motion becomes

$$
-6 \pi v a x^{(p-2)}\left(U-U_{\mathrm{sw}}\right)=m_{\mathrm{cme}} U \cdot \frac{\mathrm{d} U}{\mathrm{~d} x}
$$

and the solution is

$$
-\frac{6 \pi v a}{m_{\mathrm{cme}}(p-1)}\left[x^{p-1}-x_{0}^{p-1}\right]=U+U_{\mathrm{sw}} \ln \frac{\left(U-U_{\mathrm{sw}}\right)}{\left(U_{0}-U_{\mathrm{sw}}\right)}-U_{0} .
$$

Finally, the behavior of the speed versus position for this case can be seen in Fig. 4, where we have plotted the solution (Eq. (17)) for different initial speeds and using a value of $5 \times 10^{20} \mathrm{~cm}^{2} / \mathrm{s}$ (continuous lines) and $1.25 \times 10^{21} \mathrm{~cm}^{2} / \mathrm{s}$ (dotted lines) for the kinematic viscosity.

\section{Discussion and conclusion}

The acceleration in the interplanetary medium of CMEs is well-established (Gopalswamy et al. 2000, 2001; Manoharan et al. 2001; Manoharan 2006; Howard 2007). This acceleration has been explained by an increase of the ICME mass (snowplough model Tappin 2006) or due to Lorentz force (Chen 1996; Howard 2007), although it is more common to attribute this acceleration to drag forces (Vršnak 2001; Cargill 2004). However, the exact form, the magnitude of the related coefficients and the dependence of this drag force with the CME or SW parameters are still unknown. Even more, there are doubts about this force acting on ICMEs (Reiner et al. 2003; Forbes et al. 2006). As pointed out by Vršnak et al. (2006), by considering the kinetic energy of ICMEs, the gravity and Lorentz forces are negligible in the interplanetary space. Therefore, a drag force should act in the interchange of momentum between the ICME and the SW. All forces, Lorentz, aerodynamic drag, and gravity, decrease with the heliocentric distance (Chen 1993, 1996; Sheeley et al. 1997; Vršnak et al. 2006). Although, the Lorentz force is the most important at low heights then the drag becomes predominant after $\sim 30 R_{\odot}$ (Vršnak et al. 2006). Recently, to explain IPS and white light observations (SMEI), Howard (2007) has suggested that the drag force overcome the Lorentz force

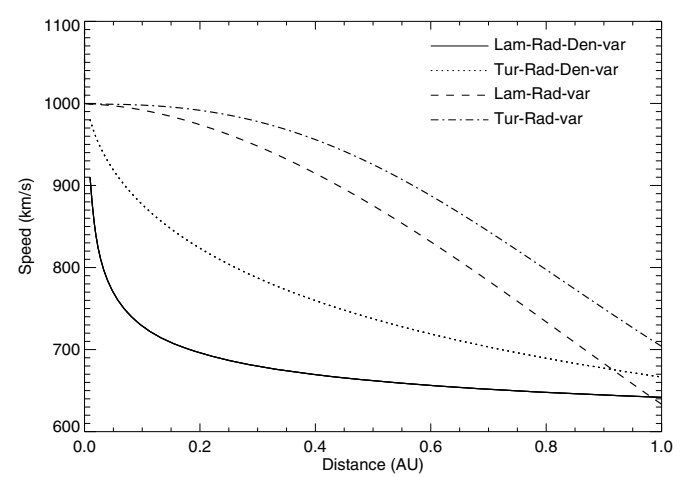

Fig. 5. ICME speed versus distance for the four models analyzed in this work. a) "laminar" regime considering variability in ICME radius (Eq. (10)) and $\mu=0.175 \mathrm{~g} / \mathrm{cm} \mathrm{s}$ (dashed line). b) "turbulent" regime considering variability in ICME radius (Eq. (11)) and $C_{\mathrm{d}}=5 \times 10^{4}$ (dot-dashed line). c) "laminar" regime considering variability in ICME radius and SW density (Eq. 17) and $v=8.75 \times 10^{20} \mathrm{~cm}^{2} / \mathrm{s}$ (continuous line). and d) "turbulent" regime considering variability in ICME radius and SW density (Eq. (14)) and $C_{\mathrm{d}}=1.1 \times 10^{5}$ (dot line).

at greater distances, between 80 to $100 R_{\odot}$. Here, since we are not taking the magnetic field effects into account, we neglect the Lorentz force. In order to understand and model the drag force acting on the ICME-SW system, we explore two forms of this force (linearly and quadratically dependent with the ICME speed) and variations in two parameters of the system (the CME radius and the SW density). Our analytical analysis helps to physically understand the effects of these parameters and dependences in the ICME dynamics. To perform such comparison, in Fig. 5 we plot Eqs. (10), (11), (14), and (17), assuming an initial speed of $1000 \mathrm{~km} \mathrm{~s}^{-1}$ and the parameters $\left(\mu\right.$ and $C_{\mathrm{d}}$ ) corresponding to the mean values of these parameters used in Figs. 1 to 4. Upon inspection of Fig. 5, it is easy to see a completely different behavior between the models considering only variability of the ICME radius and considering variability of both, ICME radius and solar wind density.

When the dominant variation is the CME radius (as $x^{0.78}$ ), the curves have negative concavity; when the density variation (as $x^{-2}$ ) is dominant, the concavity changes, meaning that the density of the medium plays an important role in the transport of the ICMEs (Reiner et al. 2003). Using scintillation data (IPS), Manoharan et al. (2001) and Manoharan (2006) have found that ICMEs show a low decline in speed below $100 R_{\odot}(0.36 \mathrm{AU})$ and a rapid decrease after this distance. This behavior agree with the concavity of the curves in Figs. 1 and 2. By analyzing type II bursts, Reiner et al. (2003) find that a drag force cannot explain the observed frequency drift. They argue that a change in the concavity of the velocity-distance curve is necessary in order to fit the type II data. We note that Reiner et al. (2003) used similar variations for both CME area $\left(\sim x^{2}\right)$ and SW density $\left(\sim x^{-2}\right)$. Here we show that it is necessary to determine the dominant parameter in the drag force in order to determine the concavity of the curve. Considering the "turbulent" regime and using the variation in radius as the dominant factor, we note the dragcoefficient between $0.6 \times 10^{5}-1.6 \times 10^{5}$; on the other hand, if we consider the density variation as the dominant factor, the drag's coefficient values diminish to the $2 \times 10^{4}-8 \times 10^{4}$ range.

Acknowledgements. A. Borgazzi acknowledges grant CNPq-Brazil. A. Lara acknowledges UNAM-PAPIIT grant IN117309 and CONACyT grants 49395 and 42577 for partial support. E. Echer would like to thank the Brazilian $\mathrm{CNPq}$ (PQ-300104/2005-7 and 470706/2006-6) agencies for financial support. M.V. Alves thanks the support given by CNPq (304865/2007-9). 


\section{References}

Batchelor, G. K. 2000, An Introduction to fluid dynamics (Cambridge University Press)

Borgazzi, A., Lara, A., Romero-Salazar, L., \& Ventura, A. 2008, Geof. Int., 47, 301

Canto, J., Gonzalez, R. F., Raga, A. C., et al. 2005, MNRAS, 357, 572

Chen, J. 1996, J. Geophys. Res., 101, A12, 27499

Chen, J., \& Garren, D. 1993, Geophys. Res. Lett., 20, 21, 2319

Cargill, P. J. 2004, Sol. Phys., 221, 135

Cargill, P. J., \& Chen, J. 1996, J. Geophys. Res., 101, A3, 4855

Daugherty, R., Franzini, J., \& Finnemore, E. 1989, Fluid Mechanics with Engineering Applications (McGraw-Hill Book Company)

Forbes, T. G., Linker, J. A., Chen, J., et al. 2006, Space Sc. Rev., 123, 1, 251

Gonzalez-Esparza, A., Lara, A., Perez-Tijerina, E., Santillan, A., \& Gopalswamy, N. 2003, J. Geophys. Res., 108, A1

Gopalswamy, N., Lara, A., Lepping, R. P., et al. 2000, Geophys. Res. Lett., 27, 145

Gopalswamy, N., Lara, A., Yashiro, S., Kaiser, M. L., \& Russell, H. 2001, J. Geophys. Res., 18, 29.207

Gopalswamy, N., Lara, A., Manoharan, P., \& Howard, R. 2005, Adv. Space Res., 36,2289

Gosling, J. T., McComas D. J., Phillips, J. L., \& Bame, S. J. 1999, J. Geophys. Res., 96, A5, 7831

Howard, T., Fry, C., \& Webb, D. 2007, ApJ., 667, 610
Kundu, P., \& Cohen, I. 2004, Fluid Mechanics (Elsevier Academic Press), third edition

Landau, L., \& Lifshitz, M. 1987, Fluid Mechanics (Pergamon Press), second edition

Leblanc, Y., Dulk, G., \& Bougeret, J. 1996, Sol. Phys., 183, 165

Liu, I., Richardson, J. D., \& Belcher, J. W. 2005, Planetary and Space Science, 53,3

Manoharan, P. 2006, Sol. Phys., 235, 345

Manoharan, P., Tokumaru, M., Pick, M., et al. 2001, ApJ, 559, 1180

Odstrčil, D., \& Pizzo, V. J. 1999a, J. Geophys. Res., 104, A1, 483

Odstrčil, D., \& Pizzo, V. J. 1999b, J. Geophys. Res., 104, A1, 493

Reiner, M. J., Kaiser, M. L., \& Bougeret, J. L. 2003, in Solar Wind Ten, ed. M. Velli, et al., AIP Conf. Proc., 679, 152

St. Cyr, O. C., Burkepile, J. T., Hundhausen, A. J., \& Lecinski, A. R. 1999, J. Geophys. Res., 104 ,A6, 12493

Sheeley, N. R., Wang, Jr., Hawley, S. H., et al. 1997, ApJ, 484, 472

Tappin, S. 2006, Sol. Phys., 233, 233

Tappin, S., Buffington, A., Cooke, C., et al. 2006, Geophys. Res. Lett., 31, L02802

Vandas, M., Fisher, S., Dryer, M., Smith, M., \& Detman, T. 1995, J. Geophys. Res., 100, A7, 12258

Vršnak, B. 2001, J. Geophys. Res., 106, A11, 25249

Vršnak, B. 2006, Adv. Space Res., 38, 431

Vršnak, B., \& Gopalswamy, N. 2002, J. Geophys. Res., 107, A2

Vršnak, B., \& Žic, T. 2007, A\&A, 472, 937

Vršnak, B., Ruždjak, D., Sudar, D., \& Gopalswamy, N. 2004, A\&A, 423, 717 